\title{
A surface with discontinuous isoperimetric profile and expander manifolds
}

\author{
Panos Papasoglu ${ }^{1}$ [D Eric Swenson ${ }^{2}$ \\ Received: 24 August 2018 / Accepted: 26 July 2019 / Published online: 3 August 2019 \\ (c) The Author(s) 2019
}

\begin{abstract}
We construct sequences of 'expander manifolds' and we use them to show that there is a complete connected 2-dimensional Riemannian manifold with discontinuous isoperimetric profile, answering a question of Nardulli and Pansu. Using expander manifolds in dimension 3 we show that for any $\epsilon, M>0$ there is a Riemannian 3-sphere $S$ of volume 1 , such that any (not necessarily connected) surface separating $S$ in two regions of volume greater than $\epsilon$, has area greater than $M$.
\end{abstract}

Keywords Isoperimetric profile · Expander · Isoperimetric inequality

Mathematics Subject Classification (1991) 53C20 - 49Q20 · 05C40

\section{Introduction}

Expander graphs have been studied widely and they have important applications both in pure mathematics and in computer science. One may construct expander-like Riemannian manifolds with interesting properties (see e.g. [3-5]). We will treat two instances of such manifolds in this paper. We think it might be useful to give a formal definition of 'expander manifolds':

Definition Let $M_{n}^{k}$ be a sequence of closed $k$-dimensional Riemannian manifolds all of which have volume 1 . We say that it is an expander sequence (or an expander manifold) if for any $n$ any smooth hypersurface $S_{n}^{k-1}$ separating $M_{n}^{k}$ in two open sets both of which have volume at least $1 / n$, has $(k-1)$-volume at least equal to $n$.

\footnotetext{
$\bowtie$ Panos Papasoglu

papazoglou@maths.ox.ac.uk

Eric Swenson

eric@math.byu.edu

1 Mathematical Institute, University of Oxford, Andrew Wiles Building, Woodstock Rd, Oxford OX2 $6 \mathrm{GG}, \mathrm{UK}$

2 Mathematics Department, Brigham Young University, Provo, UT 84602, USA
} 
Of course there are variations of this concept: e.g. one could allow manifolds with boundary or require that all $M_{n}^{k}$ are homeomorphic to a given manifold or share a common geometric property.

If $\left(M^{n}, g\right)$ is a Riemannian manifold the isoperimetric profile function of $M^{n}$ is a function $I_{M}:(0, \operatorname{vol}(M)) \rightarrow \mathbb{R}^{+}$defined by:

$$
I_{M}(t)=\inf _{\Omega}\left\{\operatorname{vol}_{n-1}(\partial \Omega): \Omega \subset M^{n}, \operatorname{vol}_{n}(\Omega)=t\right\}
$$

where $\Omega$ ranges over all open sets of $M^{n}$ with smooth boundary. We note that we don't require $\Omega$ to be connected.

It is easy to see that $I_{M}(t)$ is upper semi-continuous by adding or taking away a small ball with smooth boundary from a given region. More precisely to show upper continuity it suffices to show that for any $t_{0} \in(0, \operatorname{vol}(M))$ and $\delta>0$, there is an $\epsilon>0$ such that $I_{M}(t)<I_{M}\left(t_{0}\right)+\delta$ if $\left|t-t_{0}\right|<\epsilon$. Given some open set $\Omega$ with $\operatorname{vol}_{n}(\Omega)=t_{0}$ and $\operatorname{vol}_{n-1}(\partial \Omega)<I_{M}\left(t_{0}\right)+\frac{\delta}{2}$ we may pick $\epsilon$ small enough such that for any $\epsilon^{\prime}<\epsilon$ there are 2 small open balls with smooth boundary $B_{1}, B_{2}$ with $B_{1} \subseteq \Omega$ and $B_{2}$ contained in the complement of $\Omega$ with $\operatorname{vol}_{n}\left(B_{1}\right)=\operatorname{vol}_{n}\left(B_{2}\right)=\epsilon^{\prime}$ and $\operatorname{vol}_{n-1}\left(\partial B_{1}\right), \operatorname{vol}_{n-1}\left(\partial B_{2}\right)<\delta / 4$. Then by considering the open sets $\Omega \backslash B_{1}, \Omega \cup B_{2}$ we see that $I_{M}(t)<I_{M}\left(t_{0}\right)+\delta$ if $\left|t-t_{0}\right|=\epsilon^{\prime}$.

The continuity of the isoperimetric profile function is established in a number of cases: Gallot [9] showed that it is continuous for compact manifolds and Nardulli-Russo [16] showed that this holds also for manifolds of finite volume. Ritoré [21] showed that $I_{M}$ is continuous for Hadamard manifolds and complete non-compact manifolds of strictly positive sectional curvature. We refer to [21] and [15] for a more complete exposition of the cases where the continuity of $I_{M}$ is established and related questions.

Adams, Morgan and Nardulli observed that $I_{M}(t)$ is not necessarily continuous for manifolds with density (see Frank Morgan's blog, [1]).

Nardulli and Pansu [15] constructed an example of a Riemannian 3-manifold with discontinuous $I_{M}(t)$ and asked whether there is such an example in dimension 2.

It turns out that all these constructions are based on the existence of expander manifolds, so we answer this question in Sect. 2 of this paper by constructing an appropriate sequence of 'expander surfaces' using expander graphs. We note that P. Buser [5] has used in the past expander graphs as 'blueprints' for constructing surfaces. Psaltis [20] used expander graphs to show that there are surfaces $M$ with no restrictions on the growth rate of $I_{M}$.

The first author asked in [17] whether there are expander manifolds $M_{n}^{3}$ where each $M_{n}^{3}$ is homeomorphic to the 3-sphere (or to the 3-ball). It turns out that such constructions were already given by Burago-Ivanov in [4].

Glynn-Adey and Zhu show in [10] that for any $\epsilon>0, M>0$ there is a Riemannian 3-ball $B$ of volume 1 such that any smooth disk separating $B$ in two regions of volume greater than $\epsilon$ has area greater than $M$. We prove the same result here both for the 3-ball and the 3-sphere for separations by arbitrary surfaces and not just disks. Glynn-Adey and Zhu assume further that the ball $B$ has bounded diameter and boundary surface area but these are properties that are easy to arrange in general modifying slightly the ball $B$. Our construction is different from the one in [4], for example our sequence of manifolds converges to a point.

These results contrast with the situation in dimension 2. Liokumovich, Nabutovsky and Rotman showed in [13] that if $D$ is a Riemannian 2-disc there is a simple arc of length less than $2 \sqrt{3} \sqrt{\operatorname{area}(D)}+\delta$ which cuts the disc into two regions of area greater than area $(D) / 4-\delta$ where $\delta$ is any positive real. A similar result was shown in [18] for the sphere. The results in [13] were prompted by a question of Gromov [11] and Frankel-Katz [8] concerning bounding the length of contracting homotopies of a 2-disk. 
Balacheff-Sabourau [2] showed that there is some $c>0$ such that any Riemannian surface $M$ of genus $g$ can be separated in two domains of equal area by a 1-cycle of length smaller than $c \sqrt{g+1} \sqrt{\operatorname{area}(M)}$. Liokumovich [14] on the other hand showed that given $C>0$ and a closed surface $M$ there is a Riemannian metric of diameter 1 on $M$ such that any 1-cycle splitting it into two regions of equal area has length greater than $C$.

We thank the referee for a careful reading of the paper and several suggestions that improved the exposition.

\section{Surfaces with discontinuous profile}

We follow the same method as [1,15]. Given some constants $a, b>0$ it is enough to find a sequence of closed surfaces $S_{n}$ with area $\left(S_{n}\right)=a+\tau_{n}$ where $\tau_{n} \rightarrow 0$ such that $I_{S_{n}}(a)=I_{S_{n}}\left(\tau_{n}\right)>b$. Indeed we may join the surfaces $S_{n}$ by tubes of negligible area to obtain a surface $S$ such that $I_{S}\left(a+\tau_{n}\right) \rightarrow 0$ but $I_{S}(a)>b$.

We explain now how to construct $S_{n}$.

Our construction relies on the existence of expander graphs. We recall the definition of expanders. Let $\Gamma=(V, E)$ be a graph. For $S, T \subseteq V$ denote the set of all edges between $S$ and $T$ by

$$
E(S, T)=\{(u, v): u \in S, v \in T,(u, v) \in E\} .
$$

Recall that a graph is called $k$-regular if every vertex is contained in exactly $k$ distinct edges.

Definition The edge boundary of a set $S \subseteq V$, denoted $\partial S$ is defined as $\partial S=E\left(S, S^{c}\right)$. A $k$-regular graph $\Gamma=(V, E)$ is called a $c$-expander graph if for all $S \subset V$ with $|S| \leq|V| / 2$, $|\partial S| \geq c|S|$.

Pinsker [19] (see also [12], sec. 2.2) has shown that there is a $c>0$ such that for any $n$ large enough there is a 3 -regular $c$-expander graph with $n$-vertices.

In the course of the proof that follows we will need to show several inequalities; as obtaining best constants is irrelevant for our construction we will always be using inequalities that are easy to state and verify, rather than optimal ones. Although we could use any family of $k$-regular expander graphs it is somewhat easier to describe our examples using 3-regular expander graphs.

Consider a 3-regular $c$-expander graph $\Gamma_{n}$ with $n^{2}+n$ vertices. We give a way to replace this graph by a Riemannian surface. For each vertex $v$ we pick a Euclidean 3-sphere $S_{v}$ of radius $1 / n$. Recall that the area of this sphere is $4 \pi(1 / n)^{2}$. If $l$ is a great circle of $S_{v}$ we pick 3 equidistant points $e_{1}, e_{2}, e_{3}$ on $l$ and we consider 3 spherical caps $D_{v}^{1}, D_{v}^{2}, D_{v}^{3}$ on $S_{v}$ with centers $e_{1}, e_{2}, e_{3}$ and heights equal to $\frac{1}{10 n}$. Clearly these spherical caps are disjoint and it is easy to see that the distance between any two of them on the sphere is greater than $1 / n$.

Indeed if $D_{v}^{i}$ intersects $l$ at $x_{i}, x_{i}^{\prime}$ and $\theta=\angle x_{i} O e_{i}$ (where $O$ is the center of $S_{v}$ ) then $\cos \theta=9 / 10>\cos (\pi / 6)$ so the angle $\angle x_{i} O x_{j}$ is greater than $\pi / 3$ hence $d\left(x_{i}, x_{j}\right)>1 / n$ on the sphere (for $i \neq j$ ).

Let $C_{v}^{1}, C_{v}^{2}, C_{v}^{3}$ be the boundary curves of $D_{v}^{1}, D_{v}^{2}, D_{v}^{3}$ respectively. We note that

$$
\text { length }\left(C_{v}^{i}\right) \geq 1 / n
$$


We remove the open spherical caps $D_{v}^{1}, D_{v}^{2}, D_{v}^{3}$ from $S_{v}$ and we still denote this sphere with holes by $S_{v}$ to keep notation simple. We note that

$$
\operatorname{area}\left(S_{v}\right)=\frac{4 \pi}{n^{2}}-3 \frac{2 \pi}{10 n^{2}}=\frac{17 \pi}{5 n^{2}} .
$$

We set $a=\frac{17 \pi}{5}$, so area $\left(S_{v}\right)=a / n^{2}$.

Now to each edge $E_{i}(i=1,2,3)$ in $\Gamma$ leaving $v$ we associate the boundary curve $C_{v}^{i}$. If an edge $e$ joins the vertices $v, w$ of $\Gamma$ we identify the corresponding boundary curves of the spheres with holes $S_{v}, S_{w}$ by an isometry. This gluing produces a closed surface $S_{n}$ with

$$
\operatorname{area}\left(S_{n}\right)=\frac{a\left(n^{2}+n\right)}{n^{2}}=a+\frac{a}{n} .
$$

We set $\tau_{n}=\frac{a}{n}$.

We note that $S_{n}$ is not a smooth manifold but it is easy to slightly modify the metric to make it smooth and this modification with have a negligible effect on the following calculations, so we will pretend for the moment that $S_{n}$ is a smooth manifold. We set $b=\min \left(\frac{1}{10}, \frac{c}{4}\right)$.

Proposition 2.1 Let $\Omega$ be an open set with smooth boundary in $S_{n}$ with $\operatorname{area}(\Omega)=\tau_{n}$. Then

$$
\text { length }(\partial \Omega) \geq b \text {. }
$$

Proof We distinguish two types of spheres with holes $S_{v}$.

Type 1: length $\left(\Omega \cap\left(C_{v}^{1} \cup C_{v}^{2} \cup C_{v}^{3}\right)\right) \geq \frac{5}{2}$ length $\left(C_{v}^{1}\right)$ and

Type 2: where the opposite inequality holds.

Let's denote by $\Omega_{1}$ the intersection of $\Omega$ with all type 1 spheres and $\Omega_{2}$ its intersection with type 2 spheres.

Lemma 2.2 Let $S_{v}$ be a type 2 sphere and let $\Omega_{v}=\Omega \cap S_{v}$ and $\delta \Omega_{v}=\partial \Omega \cap S_{v}$. We have then

$$
\operatorname{area}\left(\Omega_{v}\right) \leq \frac{8 \pi}{n} \text { length }\left(\delta \Omega_{v}\right)
$$

Proof Note that area $\Omega_{v} \leq$ area $S_{v}=a / n^{2}$. We note also that $\delta \Omega_{v}$ is in fact the boundary of $\Omega_{v}$ if we consider it to be a subset of $S_{v}$ rather than $S$-for consistency's sake and to avoid ambiguity we will always consider our domains to be subsets of $S$.

We remark that our definition ensures that $\delta \Omega_{v} \subseteq \partial \Omega$ which is convenient in what follows.

Clearly it suffices to prove the inequality for all connected components $U$ of $\Omega_{v}$, namely it suffices to show:

$$
\operatorname{area}(U) \leq \frac{8 \pi}{n} \text { length }\left(\delta \Omega_{v} \cap \partial U\right)
$$

We consider the connected components of $\delta \Omega_{v}$. If there is a connected component $\alpha$ that is an arc with endpoints in two distinct $C_{v}^{i}, C_{v}^{j}$ then length $(\alpha) \geq 1 / n$ so the lemma holds as

$$
\frac{a}{n^{2}} \leq \frac{8 \pi}{n} \frac{1}{n} .
$$

Similarly if there is a connected component $\alpha$ that is a simple closed curve such that for some $i \neq j C_{v}^{i}, C_{v}^{j}$ lie in the closures of distinct connected components of $S_{v} \backslash \alpha$ then 
Fig. 1 Erasing the 'inner' arcs

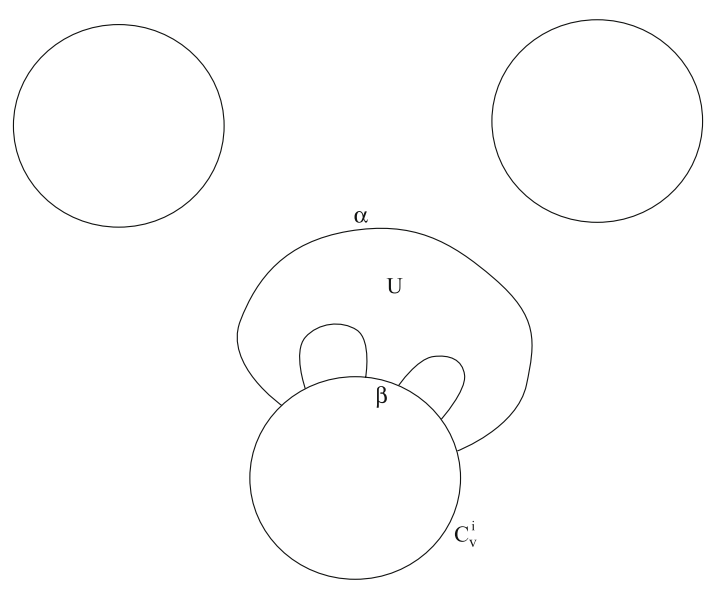

length $(\alpha) \geq 1 / n$ and the lemma holds. So we may assume that $\delta \Omega_{v}$ contains no such simple closed curve $\alpha$.

Note also that if there is a connected component of $\delta \Omega_{v}, \alpha$, which is a simple closed curve that bounds a disc $D$ in $S_{v}$ which is not contained entirely in $\Omega_{v}$ then we may delete $\alpha$. This will increase area $\left(\Omega_{v}\right)$ and decrease length $\left(\delta \Omega_{v}\right)$. So it suffices to prove the lemma for regions $\Omega_{v}$ such that $\delta \Omega_{v}$ contains no such simple closed curves.

Similarly suppose that there is a connected component $U$ of $\Omega_{v}$ such that $\partial U \cap \delta \Omega_{v}$ contains an $\operatorname{arc} \alpha$ with its endpoints on some $C_{v}^{i}$ so that if $\beta$ is the $\operatorname{arc}$ with the same endpoints on $C_{v}^{i}$, $\alpha \cup \beta$ bounds a disc on $S_{v}$ which contains $U$. In this case if we erase all arcs in $\partial U \cap \delta \Omega_{v}$ except $\alpha$ the area of $U$ increases while the boundary length decreases (see Fig. 1). So we may assume for the purposes of showing $(*)$ that if a region $U$ intersects exactly one of the $C_{v}^{i}$ then $\partial U \cap \delta \Omega_{v}$ is a single arc.

To summarize if a connected component $U$ of $\Omega_{v}$ has a simple closed curve $\alpha$ in its boundary then we may assume that $U$ is a disc with boundary $\alpha$. Also if some connected component $U$ of $\Omega_{v}$ intersects only one $C_{v}^{i}$ then we may assume (for the purpose of showing (*)) that $\partial U \cap \delta \Omega_{v}$ is a single arc. Finally if some connected component $U$ of $\Omega_{v}$ intersects two $C_{v}^{i}$,s then it intersects all $C_{v}^{1}, C_{v}^{2}, C_{v}^{3}$.

From these observations we conclude that it is enough to show $(*)$ assuming that the connected components $U$ of $\Omega_{v}$ fall in the following 3 cases (see Fig. 2):

1. Regions $U$ homeomorphic to a disc with $\partial U \subseteq \delta \Omega_{v}$. By the isoperimetric inequality of the sphere the minimal boundary length for such regions is for spherical caps. However the corresponding spherical cap has area less than the sphere with holes $S_{v}$ so it has height $h$ less than $\frac{19}{10 n}$. Recall that the area of a spherical cap is $2 \pi r h$ where $r$ is the radius of the sphere and $h$ the height. It follows that the length of the boundary of the spherical cap is greater or equal to $h / 2$ so

$$
\text { area } U \leq \frac{2 \pi h}{n} \leq \frac{4 \pi \text { length }(\partial U)}{n}
$$

and the inequality holds.

2. Regions $U$ homeomorphic to a disc with $\partial U=\alpha \cup \beta$ where $\alpha, \beta$ are arcs with the same endpoints and $\alpha \subseteq \delta \Omega_{v}, \beta \subseteq C_{v}^{i}$ for some $i$. Then clearly length $(\alpha)>$ length $(\beta)$ and 
Fig. 2 The three cases

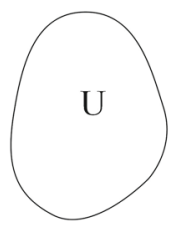

Case 1

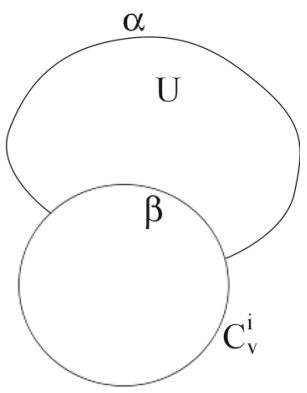

Case 2

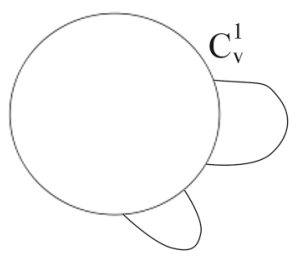

$\mathrm{U}$
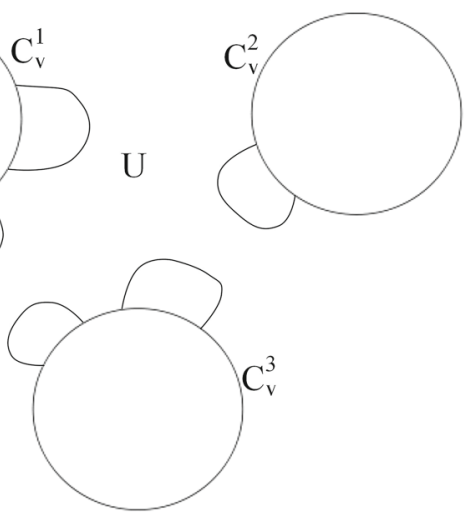

Case 3

applying the isoperimetric inequality of the sphere again we have

$$
\operatorname{area}(U) \leq \frac{8 \pi \text { length }(\partial U)}{n}
$$

and the inequality holds.

3. Regions $U$ homeomorphic to a sphere with 3 holes and $\partial U=\alpha \cup \beta$ where $\alpha$ is a union of $\operatorname{arcs}$ in $\delta \Omega_{v}$ with endpoints on the $C_{v}^{i}$ 's and $\beta$ is a union of $\operatorname{arcs}$ contained in the $C_{v}^{i}$ 's. Since $S_{v}$ is of type 2 length $(\alpha) \geq \frac{1}{2 n}$ while

$$
\operatorname{area}(U) \leq \operatorname{area}\left(S_{v}\right)=\frac{a}{n^{2}}
$$

and the inequality holds in this case too.

We note that at least one of area $\left(\Omega_{1}\right)$, area $\left(\Omega_{2}\right)$ is greater than or equal to $\tau_{n} / 2$. We will show that in both cases the proposition holds.

Let's say that area $\left(\Omega_{2}\right) \geq \tau_{n} / 2$. If $a_{v}$ is the area of $S_{v} \cap \Omega_{1}$ and $\ell_{v}$ is the length of $\partial \Omega_{1} \cap S_{v}$ then

$$
\frac{\tau_{n}}{2} \leq \sum a_{v} \leq \frac{8 \pi}{n} \sum \ell_{v}
$$

so

$$
\operatorname{length}(\partial \Omega) \geq \sum \ell_{v}>\frac{1}{10} \geq b
$$


Let's say now that area $\left(\Omega_{1}\right) \geq \tau_{n} / 2$. Let $T$ be the set of vertices of $\Gamma_{n}$ that correspond to spheres with holes of type 1 . Clearly $T$ contains at least $n / 2$ vertices.

Claim $T$ contains less than $\frac{n^{2}+n}{2}$ vertices.

Proof Indeed if $T$ contains more than $\frac{n^{2}+n}{2}$ vertices then there are at least $n^{2} / 10$ vertices $v$ of type 1 such that area $\left(S_{v} \cap \Omega_{1}\right)<\operatorname{area}\left(S_{v}\right) / 2$, otherwise we would have area $\left(\Omega_{1}\right)>\tau_{n}$ which is impossible. We show now that for each sphere $S_{v}$ in $T$ for which area $\left(S_{v} \cap \Omega_{1}\right)<$ $\operatorname{area}\left(S_{v}\right) / 2$ we have that length $\left(\partial \Omega_{1} \cap S_{v}\right) \geq 1 / n$. Indeed if $\partial \Omega_{1} \cap S_{v}$ contains an arc with endpoints in distinct $C_{v}^{i}$,s this is obvious. We note also that none of the $C_{v}^{i}$, s is entirely contained in the complement of $\Omega_{1}$ as $S_{v}$ is of type 1 . So $\partial \Omega_{1} \cap S_{v}$ consists of some arcs $\alpha_{j}(j \in J)$ such that both endpoints of the $\alpha_{j}$ lie in a single $C_{v}^{i}$. If we denote by $\beta_{j}$ the shorter of the two arcs on $C_{v}^{i}$ with the same endpoints as $\alpha_{j}$ then we note that $\alpha_{j} \cup \beta_{j}$ bounds a region of $S_{v}$ homeomorphic to a disc. If this is not contained in some hemisphere then length $\left(\alpha_{j}\right) \geq 1 / n$ so length $\left(\partial \Omega_{1} \cap S_{v}\right) \geq 1 / n$. It follows by the soperimetric inequality of the sphere that length $\left(\alpha_{j}\right)+$ length $\left(\beta_{j}\right)$ is greater than the boundary length of the spherical cap bounding the same area. We note that the union

$$
\bigcup_{j \in J}\left(\alpha_{j} \cup \beta_{j}\right)
$$

bounds a region of area greater than area $\left(S_{v}\right) / 2$. It follows that

$$
\sum_{j \in J}\left(\text { length }\left(\alpha_{j}\right)+\operatorname{length}\left(\beta_{j}\right)\right) \geq 2 \text { length }\left(C_{v}^{1}\right) .
$$

Since $S_{v}$ is of type 1

$$
\sum_{j \in J} \operatorname{length}\left(\beta_{j}\right) \leq \operatorname{length}\left(C_{v}^{1}\right) / 2
$$

Hence

$$
\sum_{j \in J} \text { length }\left(\alpha_{j}\right) \geq \operatorname{length}\left(C_{v}^{1}\right) \geq 1 / n
$$

So

$$
\text { length }\left(\partial\left(\Omega_{1}\right)\right) \geq n / 10 \text {. }
$$

This proves the claim, from which the proposition clearly follows.

By our hypothesis that $\Gamma_{n}$ is a sequence of $c$-expander graphs there are at least $\frac{c n}{2}$ edges in the boundary of $T$. If $(v, w)$ is such an edge, with $v \in T$, then for some $i \in\{1,2,3\} C_{v}^{i}$ is identified to $C_{w}^{j}$ for some $j \in\{1,2,3\}$ and $S_{v}$ is of type 1 while $S_{w}$ is of type 2 . Since $S_{v}$ is of type 1

$$
\text { length }\left(C_{v}^{i} \cap \Omega\right) \geq \frac{1}{2} \text { length } C_{v}^{i}
$$

so as $C_{v}^{i}$ is identified to $C_{w}^{j}$

$$
\text { length }\left(C_{w}^{j} \cap \Omega\right) \geq \operatorname{length}\left(C_{w}^{j}\right) / 2 .
$$


On the other hand since $S_{w}$ is of type 2

$$
\sum_{i=1}^{3} \operatorname{length}\left(C_{w}^{i} \backslash \Omega\right) \geq \operatorname{length}\left(C_{w}^{j}\right) / 2 .
$$

If we set

$$
A=C_{w}^{j} \cap \Omega
$$

and $B=\left(\bigcup_{i=1}^{3} C_{w}^{i}\right) \backslash \Omega$ we have that $\partial \Omega \cap S_{w}$ separates $A$ from $B$.

We claim now that $\partial \Omega \cap S_{w}$ has length at least $1 / 2 n$.

If $\partial \Omega \cap S_{w}$ contains some simple closed curve $\alpha$ such that each region of $S_{w} \backslash \alpha$ contains some $C_{w}^{i}$ then clearly $\partial \Omega \cap S_{w}$ has length more than $1 / 2 n$. The same holds if $\partial \Omega \cap S_{w}$ contains some arc with endpoints in distinct $C_{w}^{i}$ 's so in these cases the claim is proven.

Otherwise since $\partial \Omega$ separates $A, B$ at least one of the following two holds:

1. There is a set of $\operatorname{arcs} \beta_{j}(j \in J)$ on $\partial \Omega$ with endpoints on $C_{w}^{j}$ such that there $\operatorname{are} \operatorname{arcs} \alpha_{j}$ on $C_{v}^{j}$ where $\alpha_{j}$ has the same endpoints as $\beta_{j}$,

$$
A \subseteq \bigcup_{j \in J} \alpha_{j}
$$

and for each $j \in J \alpha_{j} \cup \beta_{j}$ bounds a disc in $S_{w}$.

2. There is a set of $\operatorname{arcs} \beta_{j}(j \in J)$ on $\partial \Omega$ with endpoints on $\bigcup_{i=1}^{3} C_{w}^{i}$ such that there are $\operatorname{arcs} \alpha_{j}$ on $\bigcup_{i=1}^{3} C_{w}^{i}$ where $\alpha_{j}$ has the same endpoints as $\beta_{j}$,

$$
B \subseteq \bigcup_{j \in J} \alpha_{j}
$$

and for each $j \in J \alpha_{j} \cup \beta_{j}$ bounds a disc in $S_{w}$.

In both cases

$$
\sum_{j \in J} \text { length } \beta_{j} \geq \sum_{j \in J} \text { length } \alpha_{j} \geq \min (\text { length } A \text {, length } B) \geq 1 / 2 n \text {. }
$$

It follows that in all cases $\partial \Omega \cap S_{w}$ has length at least $1 / 2 n$. Hence

$$
\text { length }(\partial \Omega) \geq \frac{c}{4} \geq b
$$

from which the proposition follows.

\section{A sphere hard to cut}

Definition Let $B$ be a Riemannian 3-ball. If $F \subset B$ is a smoothly embedded orientable surface with boundary we say that $F$ separates $B$ if $F \cap \partial B=\partial F$.

If $F$ is a surface separating a Riemannian 3-ball $B$ we say that $F$ cuts an $\epsilon$-piece of $B$ if $B-F$ can be written as a union of two disjoint open sets $U, V$ both of which have volume greater than $\epsilon$.

We define similarly what it means for a closed surface to cut an $\epsilon$-piece of a Riemannian 3-sphere. 
Consider a 3-regular $c$-expander graph $\Gamma_{n}$ with $n^{3}$ vertices. We give a way to 'thicken' this graph, i.e. replace it by a Riemannian handlebody. For each vertex we pick a Euclidean 3-ball $B_{v}$ of radius $1 / n$. Recall that the volume of this ball is $4 \pi / 3 \cdot(1 / n)^{3}$. Let $S_{v}$ be the boundary sphere of $B_{v}$. If $l$ is an equator $S_{v}$ we pick 3 equidistant points $e_{1}, e_{2}, e_{3}$ on $l$ and we consider 3 disjoint (spherical) discs on $S_{v}$ with centers $e_{1}, e_{2}, e_{3}$ and radii equal to $1 / n$. Clearly these discs are disjoint. Now to each edge $E_{i}$ in $\Gamma$ leaving $v$ we associate the disc with center $e_{i}$. If an edge $e$ joins the vertices $v, w$ of $\Gamma$ we identify the discs of the balls $B_{v}, B_{w}$ corresponding to this edge.

In this way we obtain a handlebody $\Sigma_{n}$. Note that $\partial \Sigma_{n} \cap B_{v}$ is a pair of pants. We will refer to $B_{v}$ later on as a filled in pair of pants and we will call the discs with centers $e_{1}, e_{2}, e_{3}$ on $S_{v}$ the holes of this pair of pants. We note that the area of $S_{v}$ is $4 \pi(1 / n)^{2}$ and the area of $S_{v}$ minus the 3 spherical discs is

$$
4 \pi(1 / n)^{2}-6 \pi(1 / n)^{2}(1-\sin 0.5)=\pi(1 / n)^{2}(6 \sin 0.5-2) .
$$

By changing the metric of $\Sigma_{n}$ slightly we get a smooth handlebody, denoted still by $\Sigma_{n}$, of volume $4 \pi / 3$. Finally by gluing appropriately thickened discs to this handlebody we obtain a ball $B_{n}$. We may assume that this gluing operation changes the volume of $B_{n}$ and the area of its boundary by a negligible amount. We may pick a simple curve $\gamma$ on $\partial B_{n}$ such that every point of $\partial B_{n}$ is at distance at most $1 / n$ from $\gamma$. By gluing a thickened disk of diameter $1 / n$ and negligible volume to $\partial B_{n}$ along $\gamma$ we obtain a new ball of arbitrarily small diameter. We still denote this 3-ball by $B_{n}$. In fact it follows also directly by the properties of expander graphs that the diameter of $B_{n}$ is bounded.

We double $B_{n}$ along its boundary to obtain a 3-sphere. By changing the metric slightly along the doubling locus we may ensure that we obtain a smooth sphere $S_{n}$ of volume $8 \pi / 3$.

Theorem 3.1 Given $\epsilon, M>0$ there is some $n$ such that any surface that cuts an $\epsilon$-piece of $B_{n}\left(\right.$ or $\left.S_{n}\right)$ has area greater than $M$.

Proof We may (and will) assume that $\epsilon<1 / 100$. Let $F$ be a (not necessarily connected) surface cutting an $\epsilon$-piece of $B_{n}$. So $B_{n}-F=U_{1} \cup U_{2}$ with $U_{1}, U_{2}$ open of volume greater than $\epsilon$. We denote by $Q_{1}, Q_{2}$ the closures of $U_{1}, U_{2}$ respectively. Without loss of generality we assume that $\operatorname{vol}\left(U_{2}\right) \geq \operatorname{vol}\left(U_{1}\right)$.

We note that $B_{n}$ contains a handlebody $\Sigma_{n}$ which is a union of filled in pairs of pants $B_{v}$-one for each vertex of the graph $\Gamma_{n}$. Clearly $S_{v} \cap \partial \Sigma_{n}$ is a pair of pants with 3 holes.

Let $B_{v}$ be one such (filled in) pair of pants. Its volume is $4 \pi / 3 n^{3}$. By the solution of the isoperimetric problem for a ball [22] if a surface cuts an $\epsilon 4 \pi / 3 n^{3}$ piece of $B_{v}$ then its area is greater than $\left(4 \pi \epsilon / 3 n^{3}\right)^{2 / 3} \geq \epsilon / n^{2}$.

Let's say that for $n_{1}$ filled in pairs of pants $F$ cuts an $\epsilon / n^{3}$ piece and that for $n_{2}$ filled in pairs of pants more than $\frac{4 \pi(1-\epsilon)}{3 n^{3}}$ of their volume is contained in $U_{1}$. Since $\operatorname{vol}\left(U_{1}\right) \leq \operatorname{vol}\left(U_{2}\right)$

$$
n_{2} \leq 2 \epsilon n^{3} \leq n^{3} / 2
$$

We distinguish two cases.

Case $1 n_{1} \geq \epsilon n^{3} / 2$. Since the area of intersection of $F$ with each one of these $n_{1}$ filled in pairs of pants is greater than $\epsilon / n^{2}$ the area of $F$ is greater than $\epsilon^{2} n / 2$ which clearly tends to infinity as $n \rightarrow \infty$.

Case $2 n_{1}<\epsilon n^{3} / 2$. Since $\operatorname{vol}\left(U_{1}\right)>\epsilon$ we have that $n_{2} \geq \epsilon n^{3} / 2$. Let's denote this set of $n_{2}$-filled pairs of pants by $A$. Let $B_{v}$ be in $A$, and let $U_{v}=B_{v} \cap U_{1}$. Since

$$
\operatorname{vol}\left(U_{v}\right) \geq \frac{4 \pi(1-\epsilon)}{3 n^{3}}
$$


by the Euclidean isoperimetric inequality the boundary of $U_{v}$ has area at least

$$
\frac{4 \pi(1-\epsilon)^{2 / 3}}{n^{2}} .
$$

Since $\epsilon<1 / 100$ it follows that if the area of $F \cap B_{v}$ is less than $\epsilon / 2 n^{2}$ then $U_{1}$ intersects non-trivially all 3 holes of the filled-in pair of pants.

In fact since the area of a spherical cap is given by $2 \pi r h$ where $r$ is the radius and $h$ the height, the area of the intersection of $U_{1}$ with a hole is greater than

$$
\frac{2 \pi}{4 n^{2}}>\frac{1}{n^{2}}
$$

Let's denote by $A_{1}$ the set of filled-in pair of pants in $A$ for which the area of intersection of $F \cap B_{v}$ is more than $\epsilon / 2 n^{2}$ and let $A_{2}=A-A_{1}$. We set $k_{1}=\left|A_{1}\right|, k_{2}=\left|A_{2}\right|$ and note that

$$
k_{1}+k_{2}=n_{2} \geq \frac{\epsilon n^{3}}{2}
$$

If $k_{1} \geq \epsilon n^{3} / 4$ then we see that the area of $F$ is greater than $\epsilon^{2} n / 8$ which clearly tends to infinity as $n \rightarrow \infty$. Otherwise $k_{2} \geq \epsilon n^{3} / 4$. By the expander property (and since $k_{2} \leq n^{3} / 2$ ) the (not necessarily connected) union of filled in pairs of pants in $A_{2}, \Sigma$, has a boundary that consists of at least

$$
c k_{2} \geq \frac{c \epsilon n^{3}}{4}
$$

holes. Let $B_{v}$ be a filled-in pair of pants adjacent to one of these holes. Clearly $B_{v}$ intersects $U_{1}$. We claim that the area of $B_{v} \cap F$ is at least $\epsilon / n^{2}$. This is clear if $F$ cuts an $4 \pi \epsilon / 3 n^{3}$ piece of $B_{v}$ or if $B_{v}$ lies in $A_{1}$. If this is not the case then more than $(1-\epsilon) 4 \pi / n^{3}$ of the volume of $B_{v}$ is contained in $U_{2}$. Let $O_{v}$ be the center of $B_{v}$. Let's denote by $C_{r}$ the sphere with radius $r$ and center $O_{v}$. Let $l_{r}$ be the length of the intersection of $F$ with $C_{r}$. If $l_{r}>1 / 10 n$ for all $r$ with $1 / n>r>9 / 10 n$ then by the co-area formula $[6,3.2 .22]$ the area of $F \cap B_{v}$ is greater than $1 / 100 n^{2}>\epsilon / n^{2}$. Otherwise we consider an $r_{0} \in(9 / 10 n, 1 / n)$ for which $l_{r_{0}}$ is smaller than $1 / 10 n$. We consider the portion $F_{1}$ of $F$ between $C_{r_{0}}$ and the boundary of $B_{v}$ and we fill the holes of $F_{1}$ lying on $C_{r_{0}}$ by minimal area discs. The total area of these disks is smaller than $\frac{\pi}{100 n^{2}}$. Let's call the surface obtained this way by $F_{2}$. Note that $F_{2}$ separates $U_{1} \cap B_{v}$ from $O_{v}$. Let $f$ be the radial projection from $O_{v}$ to $C_{1}=S_{v}$. Clearly $f\left(F_{2}\right)$ contains $S_{v} \cap U_{1}$ and by inequality (*) the area of $S_{v} \cap U_{1}$ is greater than $\frac{1}{n^{2}}$. Also $f$ is Lipschitz with Lipschitz constant less than 2. So the area of $f\left(F_{2}-F_{1}\right)$ is less than $\frac{\pi}{50 n^{2}}$. It follows that the area of $F_{1}$ is greater than

$$
\frac{1}{4 n^{2}}
$$

so the area of $F \cap B_{v}$ is greater than $\epsilon / n^{2}$ in this case too.

It follows as before that the area of $F$ is at least

$$
\frac{c \epsilon n^{3}}{4} \cdot \frac{\epsilon}{n^{2}}=\frac{c \epsilon^{2} n}{4}
$$

which clearly tends to infinity as $n \rightarrow \infty$. 
The result for the 3-sphere $S_{n}$ follows immediately from $B_{n}$ as $S_{n}$ is a union of two copies of $B_{n}$ and if a surface cuts an $\epsilon$-piece of $S_{n}$ is cuts an $\epsilon / 2$ piece in one of these two copies of $B_{n}$. Finally clearly we may normalize the volume of $S_{n}, B_{n}$ to 1 .

Remark 1 In [10] it is assumed additionally that the surface area and the diameter of the ball $B_{n}$ is bounded. However both these properties are easy to arrange. As for the surface area one may excise a small ball from the 3 -sphere $S_{n}$ in the proof above and obtain a ball $B$ such that the area of $\partial B$ is arbitrarily small. By construction $B_{n}, S_{n}$ have diameter less than 1 . In fact given any ball (in any dimension $\geq 3$ ) one can easily decrease its diameter by surgery: one may cut out a thickened simple curve and glue back in a ball with small diameter. This has no effect on the volume- or separation properties of the ball. Even though we stated our result only for dimension 3 the same construction applies for spheres (balls) of any dimension $n \geq 3$.

\section{Discussion and further questions}

A general question is what geometric properties might prevent a sequence of $k$-manifolds $M_{n}^{k}$ from being an expander. For example it is easy to see that if $M_{n}^{k}$ have Ricci curvature bounded from below then $M_{n}^{k}$ can not be an expander sequence. Glyn-Adey and Zhu showed $\left[10\right.$, thm 1.6] that $M_{n}^{3}$ is not an expander sequence if the diameters and the homological filling function of the manifolds $M_{n}^{3}$ are bounded. It is not clear however whether the bound on the diameter is necessary. We recall that the 1st homological filling function of a manifold is defined by $H F_{1}(\ell)$ is defined to be the supremum of all the filling areas of cycles of length $\ell$. One may ask:

Question 4.1 Let $M_{n}^{3}$ be a sequence of 3-manifolds homeomorphic to the 3-sphere such that for all $M_{n}^{3} H F_{1}(\ell) \leq c \ell^{2}$ for a fixed $c>0$. Is it true that $M_{n}^{3}$ is not an expander sequence?

It is clear that a sequence of expander manifolds can not 'converge' (in the GromovHausdorff sense) to a manifold. Could one make precise how these manifolds 'collapse'?

Even though a lower bound on Ricci curvature rules out expanders, interestingly the question whether such manifolds have continuous isoperimeric profile is still open (see [15], question 3 and [7]).

Open Access This article is distributed under the terms of the Creative Commons Attribution 4.0 International License (http://creativecommons.org/licenses/by/4.0/), which permits unrestricted use, distribution, and reproduction in any medium, provided you give appropriate credit to the original author(s) and the source, provide a link to the Creative Commons license, and indicate if changes were made.

\section{References}

1. Adams, C., Morgan, F., Nardulli, S.: Blog-post (2013). http://sites.williams.edu/morgan/2013/07/26/ isoperimetric-profile-continuous/. Accessed 26 July 2013

2. Balacheff, F., Sabourau, S.: Diastolic and isoperimetric inequalities on surfaces. Ann. Sci. Ecole Norm. Sup. 43, 579-605 (2010)

3. Brooks, R.: The spectral geometry of towers of coverings. J. Differ. Geom. 23(1), 97-107 (1986)

4. Burago, D., Ivanov, S.: On asymptotic isoperimetric constant of tori. Geom. Funct. Anal. 8(5), 783-787 (1998)

5. Buser, P.: On the bipartition of graphs. Discrete Appl. Math. 9(1), 105-109 (1984) 
6. Federer, H.: Geometric Measure Theory. Springer, New York (1969)

7. Flores, A.M., Nardulli, S.: Continuity and differentiability properties of the isoperimetric profile in complete noncompact riemannian manifolds with bounded geometry (2015). arXiv:1404.3245

8. Frankel, S., Katz, M.: The Morse landscape of a Riemannian disc. Ann. Inst. Fourier 43(2), 503-507 (1993)

9. Gallot, S.: Inégalités isopérimétriques et analytiques sur les variétés riemanniennes. Astérisque, (163164): 5-6, 31-91, 281 (1989), 1988. On the geometry of differentiable manifolds (Rome, 1986)

10. Glynn-Adey, P., Zhu, Z.: Subdividing 3-dimensional Riemannian disks (2015). arXiv preprint arXiv: 1508.03746

11. Gromov, M.: Asymptotic invariants of infinite groups. In: Niblo, G.A., Roller, M.A. (eds.) Geometric Group Theory, v. 2, 1-295, London Mathematical Society Lecture Note Series, vol. 182. Cambridge University Press, Cambridge (1993)

12. Hoory, S., Linial, N., Wigderson, A.: Expander graphs and their applications. Bul. Am. Math. Soc. 43(4), 439-561 (2006)

13. Liokumovich, Y., Nabutovsky, A., Rotman, R.: Contracting the boundary of a Riemannian 2-disc (2012). arXiv preprint arXiv:1205.5474

14. Liokumovich, Y.: Surfaces of small diameter with large width. J. Topol. Anal. 06, 383 (2014)

15. Nardulli, S., Pansu, P.: A discontinuous isoperimetric profile for a complete Riemannian manifold (2015). arXiv:1506.04892. (to appear in Annali della Scuola Normale Superiore di Pisa)

16. Nardulli, S., Russo, F.: On the Hamilton's isoperimetric ratio in complete Riemannian manifolds of finite volume (2015). arXiv:1502.05903

17. Papasoglu, P.: Contracting thin disks (2013). arXiv preprint arXiv:1309.2967

18. Papasoglu, P.: Cheeger constants of surfaces and isoperimetric inequalities. Trans. Am. Math. Soc 361(10), 5139-5162 (2009)

19. Pinsker, M.: On the complexity of a concentrator. In: 7th International Teletraffic Conference, Stockholm, 318/1-318/4 June 1973

20. Psaltis, P.: The isoperimetric profile of infinite genus surfaces. Geom. Dedic. 149(1), 95-102 (2010)

21. Ritoré, M.: Continuity of the isoperimetric profile of a complete Riemannian manifold under sectional curvature conditions. Rev. Mat. Iberoam. 33, no. 1 (2017). arXiv:1503.07014, 2015

22. Ros, A.: The Isoperimetric Problem in Global Theory of Minimal Surfaces, Volume 2 of Clay Math Proc, pp. 175-209. American Mathematical Society, Providence, RI (2005)

Publisher's Note Springer Nature remains neutral with regard to jurisdictional claims in published maps and institutional affiliations. 